\title{
On Period of the Sequence of Fibonacci Polynomials Modulo $m$
}

\author{
İnci Gültekin ${ }^{1}$ and Yasemin Taşyurdu ${ }^{2}$ \\ ${ }^{1}$ Department of Mathematics, Faculty of Science, Ataturk University, 25240 Erzurum, Turkey \\ ${ }^{2}$ Department of Mathematics, Faculty of Science and Letters, Erzincan University, 24000 Erzincan, Turkey \\ Correspondence should be addressed to İnci Gültekin; igultekin@atauni.edu.tr
}

Received 20 November 2012; Revised 21 January 2013; Accepted 23 January 2013

Academic Editor: Binggen Zhang

Copyright (C) 2013 İ. Gültekin and Y. Taşyurdu. This is an open access article distributed under the Creative Commons Attribution License, which permits unrestricted use, distribution, and reproduction in any medium, provided the original work is properly cited.

It is shown that the sequence obtained by reducing modulo $m$ coefficient and exponent of each Fibonacci polynomials term is periodic. Also if $p$ is prime, then sequences of Fibonacci polynomial are compared with Wall numbers of Fibonacci sequences according to modulo $p$. It is found that order of cyclic group generated with $Q_{2}$ matrix $\left(\begin{array}{ll}x & 1 \\ 1 & 0\end{array}\right)$ is equal to the period of these sequences.

\section{Introduction}

In modern science there is a huge interest in the theory and application of the Fibonacci numbers. The Fibonacci numbers $F_{n}$ are the terms of the sequence $0,1,1,2,3,5, \ldots$, where $F_{n}=F_{n-1}+F_{n-2}, n \geq 2$, with the initial values $F_{0}=0$ and $F_{1}=1$. Generalized Fibonacci sequences have been intensively studied for many years and have become an interesting topic in Applied Mathematics. Fibonacci sequences and their related higher-order sequences are generally studied as sequence of integer. Polynomials can also be defined by Fibonacci-like recurrence relations. Such polynomials, called Fibonacci polynomials, were studied in 1883 by the Belgian mathematician Eugene Charles Catalan and the German mathematician E. Jacobsthal. The polynomials $F_{n}(x)$ studied by Catalan are defined by the recurrence relation

$$
F_{n}(x)=x F_{n-1}(x)+F_{n-2}(x), \quad n \geq 3,
$$

where $F_{1}(x)=1, F_{2}(x)=x$. The Fibonacci polynomials studied by Jocobstral are defined by

$$
J_{n}(x)=J_{n-1}(x)+x J_{n-2}(x), \quad n \geq 3,
$$

where $J_{1}(x)=J_{2}(x)=1$. The Fibonacci polynomials studied by P. F. Byrd are defined by

$$
\varphi_{n}(x)=2 x \varphi_{n-1}(x)+\varphi_{n-2}(x), \quad n \geq 2,
$$

where $\varphi_{0}(x)=0, \varphi_{1}(x)=1$. The Lucas polynomials $L_{n}(x)$, originally studied in 1970 by Bicknell and they are defined by

$$
L_{n}(x)=x L_{n-1}(x)+L_{n-2}(x), \quad n \geq 2,
$$

where $L_{0}(x)=2, L_{1}(x)=x[1]$.

Hoggatt and Bicknell introduced a generalized Fibonacci polynomials and their relationship to diagonals of Pascal's triangle [2]. Also after investigating the generalized Q-matrix, Ivie introduced a special case [3]. Nalli and Haukkanen introduced $h(x)$-Fibonacci polynomials that generalize both Catalan's Fibonacci polynomials and Byrd's Fibonacci Polynomials and the $k$-Fibonacci number. Also they provided properties for these $h(x)$-Fibonacci polynomials where $h(x)$ is a polynomial with real coefficients [1].

Definition 1. The Fibonacci polynomials are defined by the recurrence relation

$$
F_{n}(x)= \begin{cases}0, & \text { if } n=0, \\ 1, & \text { if } n=1, \\ x F_{n-1}(x)+F_{n-2}(x), & \text { if } n \geq 2,\end{cases}
$$

that the Fibonacci polynomials are generated by a matrix $Q_{2}$,

$$
Q_{2}=\left(\begin{array}{cc}
x & 1 \\
1 & 0
\end{array}\right), \quad Q_{2}^{n}=\left(\begin{array}{cc}
F_{n+1}(x) & F_{n}(x) \\
F_{n}(x) & F_{n-1}(x)
\end{array}\right)
$$


TABLE 1

\begin{tabular}{lllll}
\hline Fibonacci polynomials & \multicolumn{3}{c}{ Coefficient array } \\
\hline$F_{0}(x)=0$ & 0 & & \\
$F_{1}(x)=1$ & 1 & & \\
$F_{2}(x)=x$ & 1 & & & \\
$F_{3}(x)=x^{2}+1$ & 1 & 1 & & \\
$F_{4}(x)=x^{3}+2 x$ & 1 & 2 & & \\
$F_{5}(x)=x^{4}+3 x^{2}+1$ & 1 & 3 & 1 & 1 \\
$F_{6}(x)=x^{5}+4 x^{3}+3 x$ & 1 & 4 & 3 & $\vdots$ \\
$F_{7}(x)=x^{6}+5 x^{4}+6 x^{2}+1$ & 1 & 5 & 6 & $\vdots$ \\
$\vdots$ & $\vdots$ & $\vdots$ & $\vdots$ & \\
\hline
\end{tabular}

can be verified quite easily by mathematical induction. The first few Fibonacci polynomials and the array of their coefficients are shown in Table 1 [2].

A sequence is periodic if, after a certain point, it consists of only repetitions of a fixed subsequence. The number of elements in the repeating subsequence is called the period of the sequence. For example, the sequence $a, b, c$, $d, e, b, c, d, e, b, c, d, e, \ldots$, is periodic after the initial element $a$ and has period 4. A sequence is simply periodic with period $k$ if the first $k$ elements in the sequence form a repeating subsequence. For example, the sequence $a, b, c, d$, $a, b, c, d, a, b, c, d, \ldots$, is simply periodic with period 4 [4]. The minimum period length of $\left(F_{i} \bmod n\right)_{i=-\infty}^{\infty}$ sequence is stated by $k(n)$ and is named Wall number of $n$ [5].

Theorem 2. $k(n)$ is an even number for $n \geq 3$ [5].

\section{The Generalized Sequence of Fibonacci Polynomials Modulo $m$}

Reducing the generalized sequence of coefficient and exponent of each Fibonacci polynomials term by a modulus $m$, we can get a repeating sequence, denoted by

$$
\left\{F(x)^{m}\right\}=\left\{F_{0}(x)^{m}, F_{1}(x)^{m}, \ldots, F_{n}(x)^{m}, \ldots\right\},
$$

where $F_{i}(x)^{m}=F_{n}(x)(\bmod m)$. Let $h F(x)^{m}$ denote the smallest period of $\left\{F(x)^{m}\right\}$, called the period of the generalized Fibonacci polynomials modulo $m$.

Theorem 3. $\left\{F(x)^{m}\right\}$ is a periodic sequence.

Proof. Let $S_{2}=\left\{\left(x_{1}, x_{2}\right): 1 \leq x_{i} \leq 2\right\}$ where $x_{i}$ is reduction coefficient and exponent of each term in $F_{n}(x)$ polynomials modulo $m$. Then, we have $\left|S_{2}\right|=\left(m^{m}\right)^{2}$ being finite, that is, for any $i>j$, there exist natural numbers $i$ and $j$

$$
\begin{gathered}
F_{i+1}(x)^{m}=F_{j+1}(x)^{m}, \\
F_{i+2}(x)^{m}=F_{j+2}(x)^{m}, \ldots, F_{i+k}(x)^{m}=F_{j+k}(x)^{m} .
\end{gathered}
$$

By definition of the generalized Fibonacci polynomials we have that $F_{i}(x)^{m}=x F_{i-1}(x)^{m}+F_{i-2}(x)^{m}$ and $F_{j}(x)^{m}=$ $x F_{j-1}(x)^{m}+F_{j-2}(x)^{m}$. Hence, $F_{i}(x)^{m}=F_{j}(x)^{m}$, and then it follows that

$$
\begin{gathered}
F_{i-1}(x)^{m}=F_{j-1}(x)^{m} \\
F_{i-2}(x)^{m}=F_{j-2}(x)^{m}, \ldots, F_{i-j}(x)^{m}=F_{j-j}(x)^{m}=F_{0}(x)^{m}
\end{gathered}
$$

which implies that the $\left\{F(x)^{m}\right\}$ is a periodic sequence.

Example 4. For $m=2,\left\{F(x)^{2}\right\}$ sequence is $F_{0}(x)^{2}=0$, $F_{1}(x)^{2}=1, F_{2}(x)^{2}=x, F_{3}(x)^{2}=x^{2}+1=x^{0}+1=2=0$, $F_{4}(x)^{2}=0 x+x=x, F_{5}(x)^{2}=x^{2}+0=x^{0}+0=1$, $F_{6}(x)^{2}=x+x=2 x=0, F_{7}(x)^{2}=0 x+1=1$. We have $\left\{F(x)^{2}\right\}=\{0,1, x, 0, x, 1,0,1, \ldots\}$, and then repeat. So, we get $h F(x)^{2}=6$.

Given a matrix $A=\left(h_{i j}(x)\right)$ where $h_{i j}(x)$ 's being polynomials with real coefficients, $A(\bmod m)$ means that every entry of $A$ is modulo $m$, that is, $A(\bmod m)=\left(h_{i j}(x)(\bmod m)\right)$. Let $\left\langle Q_{2}\right\rangle_{m}=\left\{Q_{2}^{i}(\bmod m) \mid i \geq 0\right\}$ be a cyclic group and $\left|\left\langle Q_{2}\right\rangle_{m}\right|$ denote the order of $\left\langle Q_{2}\right\rangle_{m}$ where $Q_{2}^{i}(\bmod m)$ is reduction coefficient and exponent of each polynomial in $Q_{2}^{i}$ matrix modulo $m$.

Theorem 5. One has $h F(x)^{m}=\left|\left\langle Q_{2}\right\rangle_{m}\right|$.

Proof. Proof is completed if it is that $h F(x)^{m}$ is divisible by $\left|\left\langle Q_{2}\right\rangle_{m}\right|$ and that $\left|\left\langle Q_{2}\right\rangle_{m}\right|$ is divisible by $h F(x)^{m}$. Fibonacci polynomials are generated by a matrix $Q_{2}$,

$$
Q_{2}=\left(\begin{array}{cc}
x & 1 \\
1 & 0
\end{array}\right), \quad Q_{2}^{n}=\left(\begin{array}{cc}
F_{n+1}(x) & F_{n}(x) \\
F_{n}(x) & F_{n-1}(x)
\end{array}\right) .
$$

Thus, it is clear that $\left|\left\langle Q_{2}\right\rangle_{m}\right|$ is divisible by $h F(x)^{m}$. Then we need only to prove that $h F(x)^{m}$ is divisible by $\left|\left\langle Q_{2}\right\rangle_{m}\right|$. Let $h F(x)^{m}=t$. It is seen that $Q_{2}^{t}=\left(\begin{array}{cc}F_{t+1}(x) & F_{t}(x) \\ F_{t}(x) & F_{t-1}(x)\end{array}\right)$. Hence $Q_{2}^{t}=I(\bmod m)$. We get that $\left|\left\langle Q_{2}\right\rangle_{m}\right|$ is divisible by $t$. That is, $h F(x)^{m}$ is divisible by $\left|\left\langle Q_{2}\right\rangle_{m}\right|$. So, we get $h F(x)^{m}=$ $\left|\left\langle Q_{2}\right\rangle_{m}\right|$.

Theorem 6. $h F(x)^{p}=p k(p)$ where $p$ is a prime number.

Proof. It is completed if it is that $h F(x)^{p}$ is divisible by $p k(p)$ and that $p k(p)$ divisible by $h F(x)^{p}$. From Theorem $5 Q_{2}^{n}=$ $\left(\begin{array}{cc}F_{n+1}(x) & F_{n}(x) \\ F_{n}(x) & F_{n-1}(x)\end{array}\right), Q_{2}^{h F(x)^{p}}=I(\bmod p)$ for $Q_{2}=\left(\begin{array}{cc}x & 1 \\ 1 & 0\end{array}\right)$. Also, $Q_{2}^{p k(p)}=\left(\begin{array}{cc}F_{p k(p)+1}(x) & F_{p k(p)}(x) \\ F_{p k(p)}(x) & F_{p k(p)-1}(x)\end{array}\right)$. So, we get $Q_{2}^{p k(p)}=I(\bmod p)$. Thus $p k(p)$ is divisible by $h F(x)^{p}$. Moreover $p k(p)$ is divisible by $h F(x)^{p}$. Since $\left|\left\langle Q_{2}\right\rangle_{p}\right|=h F(x)^{p}, h F(x)^{p}$ is divisible by $p k(p)$. Therefore $h F(x)^{p}=p k(p)$.

Theorem 7. $h F(x)^{p}$ is an even number where $p$ is a prime number.

Proof. It has been shown that $h F(x)^{p}=p k(p)$ in Theorem 6. If it is stated that $p k(p)$ is an even number then proof is 
TABLE 2: Periods of the sequence of Fibonacci polynomials modulo $p$.

\begin{tabular}{lccc}
\hline$p$ & $k(p)$ & $h F(x)^{p}$ & Result \\
\hline 2 & 3 & 6 & $h F(x)^{2}=2 k(2)$ \\
7 & 16 & 112 & $h F(x)^{7}=7 k(7)$ \\
37 & 76 & 2812 & $h F(x)^{37}=37 k(37)$ \\
103 & 208 & 21424 & $h F(x)^{103}=103 k(103)$ \\
181 & 90 & 16290 & $h F(x)^{181}=181 k(181)$ \\
241 & 240 & 57840 & $h F(x)^{241}=241 k(241)$ \\
373 & 748 & 279004 & $h F(x)^{373}=373 k(373)$ \\
653 & 1308 & 854124 & $h F(x)^{653}=653 k(653)$ \\
853 & 1708 & 1456924 & $h F(x)^{853}=853 k(853)$ \\
\hline
\end{tabular}

completed. By Theorem 2, $k(p)$ is an even number and $p$ is an even number for $p \geq 3$. Hence $p k(p)$ is always an even number. That is, $h F(x)^{p}$ is an even number.

Table 2 shows some periods of sequence of coefficient and exponent of Fibonacci polynomials modulo, which is a prime number, by using $k(p)$.

\section{References}

[1] A. Nalli and P. Haukkanen, "On generalized Fibonacci and Lucas polynomials," Chaos, Solitons and Fractals, vol. 42, no. 5, pp. 3179-3186, 2009.

[2] V. E. Hoggatt Jr. and M. Bicknell, "Generalized Fibonacci polynomials and Zeckendorf's theorem," The Fibonacci Quarterly, vol. 11, no. 4, pp. 399-419, 1973.

[3] J. Ivie, "A general Q-matrix," The Fibonacci Quarterly, vol. 10, no. 3, pp. 255-261, 1972.

[4] S. W. Knox, "Fibonacci sequences in finite groups," The Fibonacci Quarterly, vol. 30, no. 2, pp. 116-120, 1992.

[5] D. D. Wall, "Fibonacci series modulo $m$," The American Mathematical Monthly, vol. 67, pp. 525-532, 1960. 


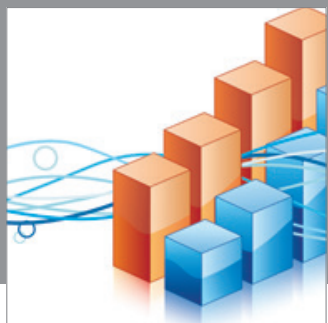

Advances in

Operations Research

mansans

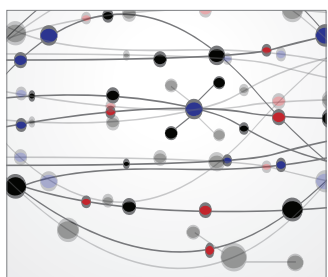

The Scientific World Journal
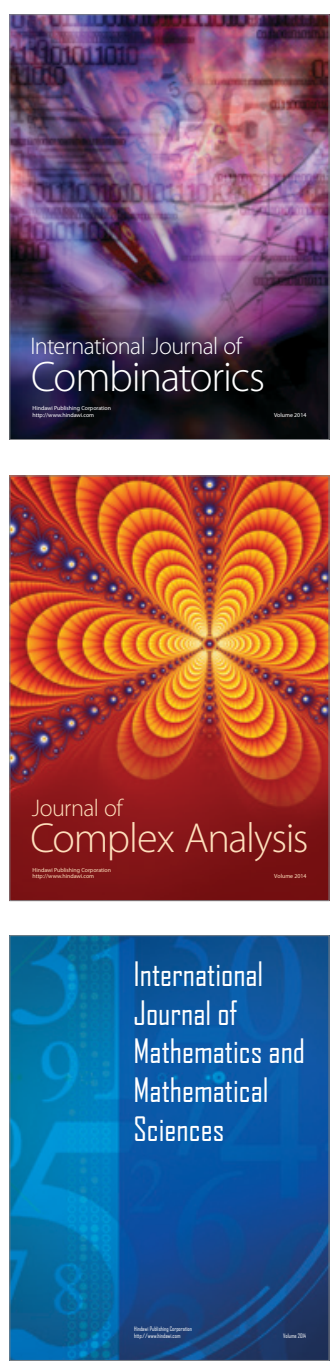
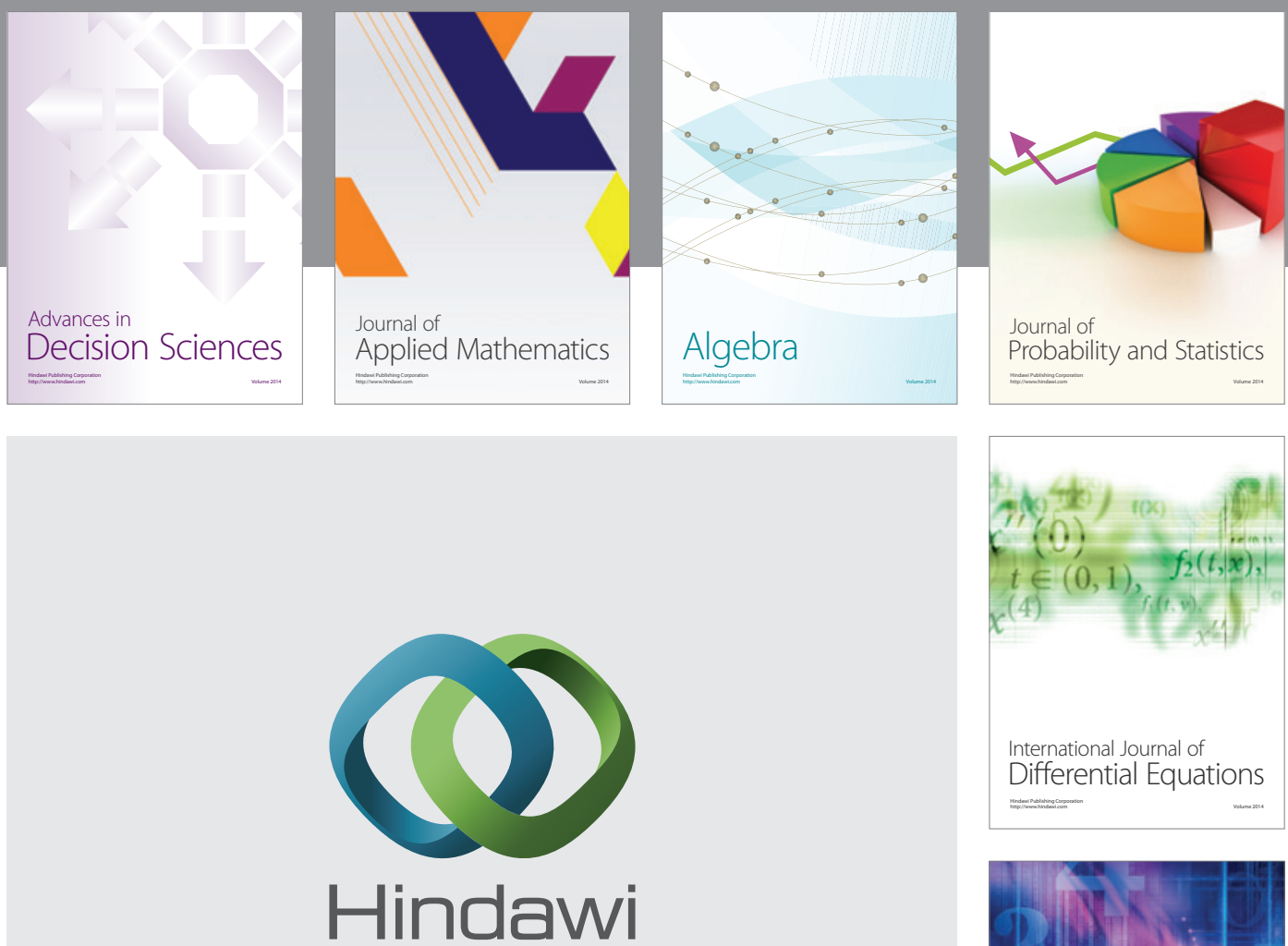

Submit your manuscripts at http://www.hindawi.com
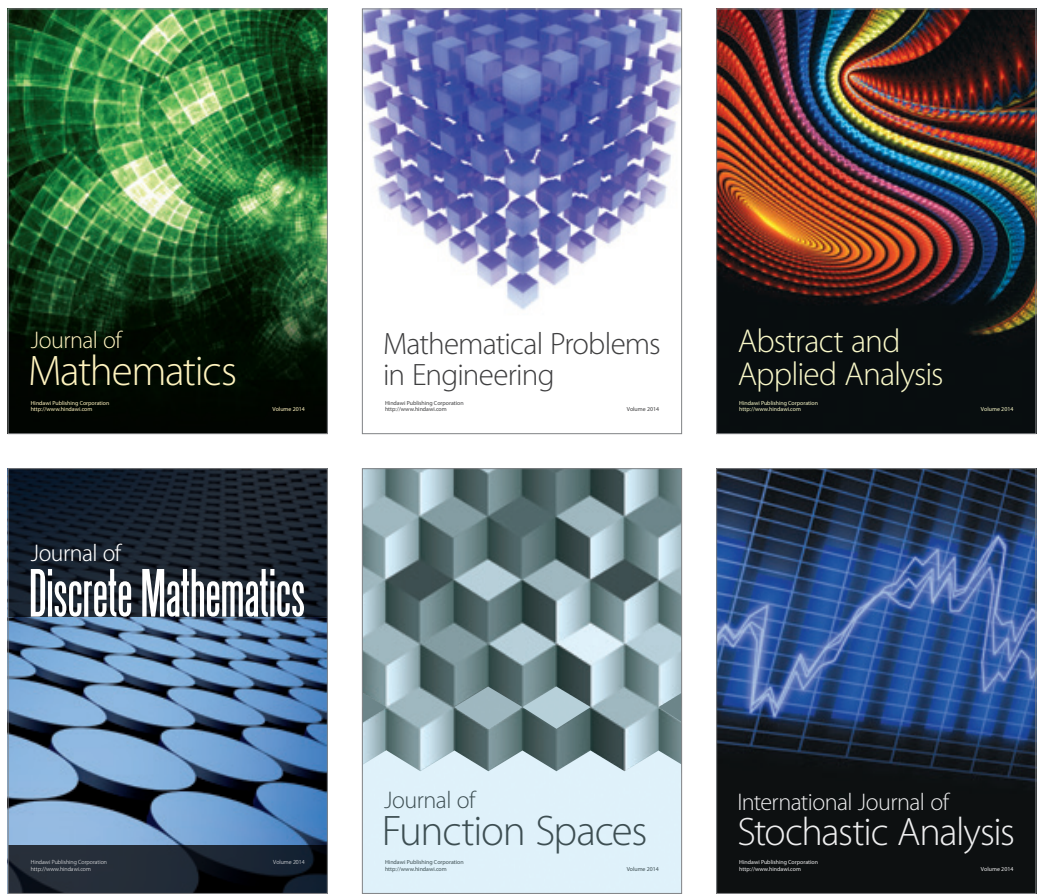

Journal of

Function Spaces

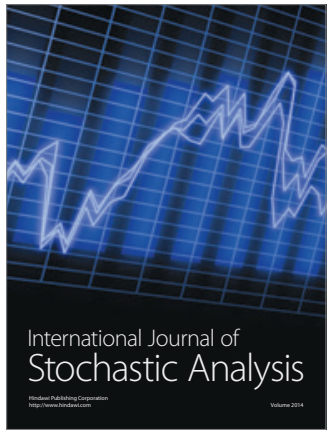

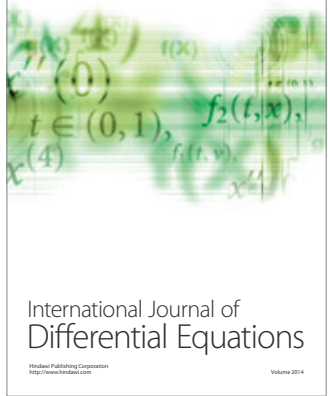
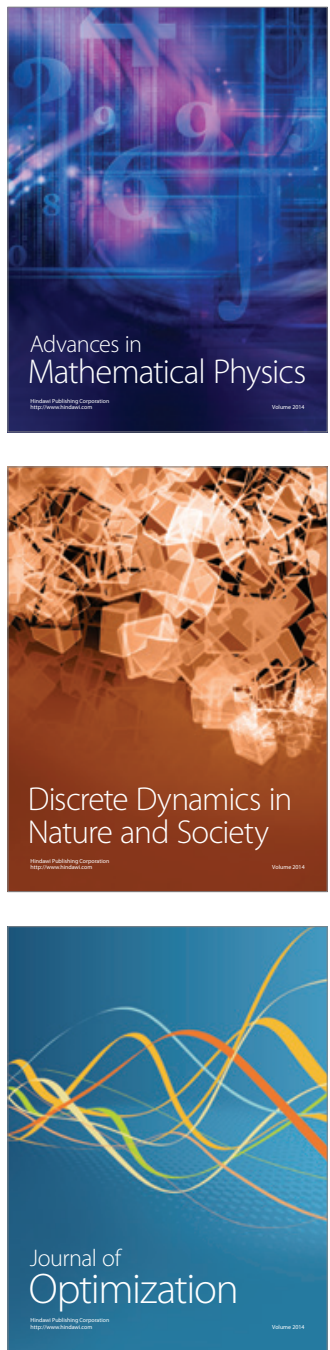